\title{
Trimethoprim-sulfamethoxazole versus vancomycin for severe infections caused by meticillin resistant Staphylococcus aureus: randomised controlled trial
}

\author{
Mical Paul,, 2 Jihad Bishara,, 2 Dafna Yahav,,,3 Elad Goldberg,,2,4 Ami Neuberger,,,6 \\ Nesrin Ghanem-Zoubi, ${ }^{7}$ Yaakov Dickstein, 6,8 William Nseir, 9 Michael Dan, ${ }^{2,10}$ Leonard Leibovici²,3
}

1Unit of Infectious Diseases,

Rabin Medical Center, Beilinson

Hospital, Petah-Tikva, Israel

${ }^{2}$ Sackler Faculty of Medicine,

Tel-Aviv University, Ramat-Aviv,

Israe

3 Unit of Infectious Diseases,

Medicine E, Rabin Medical

Center, Beilinson Hospital,

Petah-Tikva

${ }^{4} U$ nit of Infectious Diseases,

Medicine F, Rabin Medical

Center, Beilinson Hospital,

Petah-Tikva

${ }^{5}$ Division of Infectious Diseases, Medicine B, Rambam Health

Care Campus, Haifa, Israel

${ }^{6}$ Technion-Israel Institute of Technology and the Ruth \& Bruce Rappaport Faculty of

Medicine, Haifa

${ }^{7}$ Division of Infectious Diseases, Rambam Health Care Campus,

Haifa

${ }^{8}$ Division of Infectious Diseases, Medicine A, Rambam Health

Care Campus, Haifa

IInternal Medicine Department, Holy Family Hospital, Nazareth,

Faculty of Medicine in the

Galilee, Bar-Ilan Univesity,

Safed, Israel

10Infectious Diseases Unit, E Wolfson Hospital, Holon, Israel Correspondence to: Mical Paul, Division of Infectious Diseases, Rambam Health Care Campus, Haifa, 31096, Israel paulm@post.tau.ac.il

Cite this as: BMJ 2015;350:h2219 doi: 10.1136/bmj.h2219

Accepted: 20 February 2015

\section{ABSTRACT}

OBJECTIVE

To show non-inferiority of trimethoprim-

sulfamethoxazole compared with vancomycin for the

treatment of severe infections due to meticillin

resistant Staphylococcus aureus (MRSA).

DESIGN

Parallel, open label, randomised controlled trial.

SETTING

Four acute care hospitals in Israel.

\section{PARTICIPANTS}

Adults with severe infections caused by MRSA susceptible to trimethoprim-sulfamethoxazole and vancomycin. Patients with left sided endocarditis, meningitis, chronic haemodialysis, and prolonged neutropenia were excluded.

\section{INTERVENTIONS}

Trimethoprim-sulfamethoxazole $320 \mathrm{mg} / 1600 \mathrm{mg}$ twice daily versus vancomycin $1 \mathrm{~g}$ twice daily for a minimum of seven days and then by indication.

\section{MAIN OUTCOME MEASURES}

The primary efficacy outcome was treatment failure assessed at day 7, consisting of death, persistence of haemodynamic instability or fever, stable or worsening Sequential Organ Failure Assessment score, and persistence of bacteraemia. The primary safety outcome was all cause mortality at day 30 . Noninferiority was defined by a difference of less than $15 \%$ for treatment failure.

RESULTS

252 patients were included in the trial, of whom 91 (36\%) had bacteraemia. No significant difference in treatment failure was seen for trimethoprimsulfamethoxazole $(51 / 135,38 \%)$ versus vancomycin

\section{WHAT IS ALREADY KNOWN ON THIS TOPIC}

Trimethoprim-sulfamethoxazole has excellent coverage against meticillin resistant Staphylococcus aureus (MRSA) strains in most locations worldwide

Vancomycin is inferior to $\beta$ lactams for meticillin susceptible $S$ aureus and might be losing effectiveness against MRSA owing to minimum inhibitory concentration "creep" No contemporary randomised controlled trials have compared trimethoprimsulfamethoxazole with vancomycin in the treatment of severe MRSA infections

\section{WHAT THIS STUDY ADDS}

In a randomised controlled trial including 252 inpatients, trimethoprim-

sulfamethoxazole did not achieve non-inferiority to vancomycin in the treatment of severe MRSA infections

The difference was particularly marked for patients with bacteraemia

Adverse events rates were similar for trimethoprim-sulfamethoxazole and vancomycin

(32/117, 27\%)-risk ratio 1.38 (95\% confidence interval 0.96 to 1.99). However, trimethoprim-sulfamethoxazole did not meet the non-inferiority criterion-absolute difference $10.4 \%$ ( $95 \%$ confidence interval $-1.2 \%$ to $21.5 \%$ ). For patients with bacteraemia, the risk ratio was 1.40 (0.91 to 2.16). In a multivariable logistic regression analysis, trimethoprim-sulfamethoxazole was significantly associated with treatment failure (adjusted odds ratio 2.00, 1.09 to 3.65). The 30 day mortality rate was $32 / 252$ (13\%), with no significant difference between arms. Among patients with bacteraemia, 14/41 (34\%) treated with trimethoprimsulfamethoxazole and 9/50 (18\%) with vancomycin died (risk ratio 1.90, 0.92 to 3.93 ).

\section{CONCLUSIONS}

High dose trimethoprim-sulfamethoxazole did not achieve non-inferiority to vancomycin in the treatment of severe MRSA infections. The difference was particularly marked for patients with bacteraemia.

TRIAL REGISTRATION

Clinical trials NCT00427076.

\section{Introduction}

Trimethoprim-sulfamethoxazole is an old antibiotic active against Staphylococcus aureus. Trimethoprim is the main active component and bactericidal in itself, but the combination is highly synergistic. ${ }^{1}$ With increasing rates of meticillin resistant Staphylococcus aureus (MRSA) infections in healthcare settings and in the community, trimethoprim-sulfamethoxazole has been suggested as a convenient treatment option..$^{2-4}$

MRSA isolates have retained susceptibility to trimethoprim-sulfamethoxazole in many locations worldwide despite several decades of exposure to the antibiotic. Coverage rates above $90 \%$ are described in contemporary reports for community associated MRSA and nosocomial isolates in the United States, ${ }^{5-8}$ Canada, ${ }^{9-11}$ Japan, ${ }^{12}$, Europe, Israel, and Turkey. ${ }^{13-15}$ Resistance is described in Australia, where 30\% of nosocomial and $10 \%$ of community associated MRSA isolates were resistant to trimethoprim-sulfamethoxazole in 2012, but a significant trend for decreasing resistance from 2005 was observed, unlike other antibiotics. ${ }^{1617}$ In sub-Saharan Africa, 19\% resistance has been recently documented, owing to high rates of trimethoprim resistance. ${ }^{18}$ In India, more than $85 \%$ of MRSA isolates were resistant to trimethoprim-sulfamethoxazole between 2009 and 2011. ${ }^{1920}$

Trimethoprim-sulfamethoxazole is recommended for the treatment of uncomplicated skin and soft tissue infections but not for MRSA bacteraemia or pneumonia. ${ }^{21}$ 
Vancomycin is the primary treatment recommendation for these infections. Alternatives to vancomycin are sought as vancomycin is inferior to $\beta$ lactams in meticillin susceptible $S$ aureus infections, ${ }^{22}{ }^{23}$ and it may be less effective against MRSA at the higher end of the vancomycin susceptible range. ${ }^{24}$ In a small observational study, we reported similar outcomes for trimethoprim-sulfamethoxazole and vancomycin in the treatment of MRSA bacteraemia. ${ }^{25}$ Favourable outcomes for trimethoprim-sulfamethoxazole compared with vancomycin were reported in an observational study focusing on infections caused by MRSA with a minimal inhibitory concentration of $2 \mu \mathrm{g} / \mathrm{mL}$ to vancomycin. ${ }^{8}$ In both studies, treatment with trimethoprim-sulfamethoxazole was given to significantly less ill patients.

We did a randomised controlled trial to assess whether trimethoprim-sulfamethoxazole is non-inferior to vancomycin for the treatment of inpatients with severe MRSA infections, including bacteraemia.

\section{Methods}

This was an open label, parallel, one to one randomised controlled trial, conducted in four acute care hospitals (Rabin Medical Center, Petah-Tikva (192 patients); Rambam Health Care Campus (38 patients); Holy Family Hospital Nazareth (7 patients); Wolfson Medical Center (6 patients)) in Israel, between July 2007 and April 2014. Informed consent was obtained from all patients or their legal guardian.

\section{Study population and procedures}

We included adult inpatients with severe infections caused by MRSA, including bacteraemia, and patients with highly probable MRSA infections. We defined bacteraemia as the isolation of MRSA in more than one blood culture bottle or isolated in a single bottle and accompanied by fever above $38^{\circ} \mathrm{C}$, chills, or systolic blood pressure under $90 \mathrm{~mm} \mathrm{Hg}$. We defined other microbiologically documented MRSA infections by using predefined criteria adapted from surveillance definitions of healthcare associated infections, ${ }^{26}$ plus isolation of MRSA from a sterile sample from the source of infection. Patients with skin and soft tissue infections could be included only if they fulfilled the sepsis inflammatory response syndrome criteria. $^{27}$ Patients with polymicrobial infections could be included, except those involving meticillin susceptible $S$ aureus or mandating treatment with vancomycin or trimethoprim-sulfamethoxazole. The highly probable group included patients with ventilator associated pneumonia and prior antibiotic treatment, central catheter related infections, and surgical site infections in the presence of a foreign body, all without microbiological documentation. ${ }^{28}$ We excluded patients who had received trimethoprim-sulfamethoxazole or vancomycin for more than 48 hours; patients with MRSA resistant to trimethoprim-sulfamethoxazole or vancomycin; those with highly suspected or confirmed left sided endocarditis or meningitis; patients with chronic renal failure (creatinine clearance $<15 \mathrm{~mL} / \mathrm{min}$ ) and chronic hae- modialysis (those with severe acute renal failure, including acute haemodialysis, could be included); neutropenic patients with acute leukaemia or bone marrow transplantation; and patients with known allergy to either study drug, treatment with methotrexate, pregnancy or lactation, previous enrolment in this study, or concurrent participation in another trial.

We used a central computer generated random number list to randomise patients to treatment with trimethoprim-sulfamethoxazole or vancomycin. Allocation was concealed in sealed, opaque numbered envelopes that were opened consecutively after informed consent was obtained. Trimethoprim-sulfamethoxazole was started intravenously at a dose of $320 \mathrm{mg}$ trimethoprim/1600 mg sulfamethoxazole twice daily and could be switched to oral treatment using the same dose at the discretion of the treating physician. Dosing was based on a pharmacokinetic study showing that a lower dose resulted in inhibition but not killing of $S$ aureus and on the dose used in a previous trial. ${ }^{23} 29$ The vancomycin starting dose was 1 g twice daily. In both arms, treatment was adjusted to renal function; in the vancomycin arm, it was directed by serum concentrations to obtain drug trough concentrations between 10 and $20 \mathrm{mg} / \mathrm{dL}$. The complete study protocol is available on request. Treatment had to be administered for a minimum of seven days, following which the duration depended on the indication. Concomitant antibiotics, other than the combination of vancomycin and co-trimoxazole, could be administered. After randomisation, no blinding was performed, but outcomes were adjudicated blinded to allocation.

\section{Outcomes}

The primary efficacy outcome was clinical treatment failure at seven days and was a composite of death, persistence of fever $\left(<38^{\circ} \mathrm{C}\right.$ for two consecutive days and no increase above $38^{\circ}$ after resolution was required to rule out persistence), persistence of hypotension (<90 mm Hg systolic or need for vasopressor support), non-improving Sequential Organ Failure Assessment (SOFA) score (for baseline SOFA $\geq 3$, a decrease of at least $30 \%$ was required; and for baseline $S O F A<3$, a stable or decreased SOFA score was required to rule out failure), or persistent bacteraemia on day 7 . Blood cultures were repeated on day 2 for all patients with bacteraemia and daily thereafter for patients with persistent fever, persistent bacteraemia, or other signs of infection. We also defined a primary safety outcome of all cause mortality at 30 days.

Secondary outcomes included failure or modification of treatment, comprising treatment failure (as defined) or antibiotic treatment modification; bacteriological failure, defined as growth of MRSA on day 7 cultures; persistence of bacteraemia at 48 hours; length of hospital admission; duration of fever, summing all days with at least one measurement of temperature $38^{\circ} \mathrm{C}$ or above until day 30; and development of resistance defined as acquisition of trimethoprim-sulfamethoxazole or vancomycin resistant $S$ aureus or vancomycin resistant Enterococci. Adverse events included renal failure defined using RIFLE criteria, ${ }^{30}$ rash, leucopoenia, any diarrhoea and Clostridium difficile associated diarrhoea, and other adverse events. 


\section{Sample size and analysis}

To establish non-inferiority, we allowed a difference of up to $15 \%$ in the primary outcome. Assuming a $30 \%$ treatment failure rate for both treatment groups, we needed a sample of 128 patients per arm for a one sided test to rule out the pre-specified difference in the $95 \%$ confidence interval of the difference between groups, allowing for $10 \%$ non-evaluable patients $(\alpha=0.05$, $\beta=0.8$ ). We did interim analyses of the primary safety outcome after recruitment of one third and two thirds of patients, with stopping boundaries (two sided $\alpha$ level, $\mathrm{P}<0.01$ ).

We did the primary analysis by intention to treat. We did per protocol analysis for patients without exclusion criteria after randomisation who received allocated treatment for a minimum of seven days. We did subgroup analysis for patients with MRSA bacteraemia. We used $\chi^{2}$ or Fisher's exact tests to compare categorical outcomes. Continuous outcomes were skewed, so we described them as medians and compared them by using a median difference, all with $95 \%$ confidence intervals. ${ }^{31}$ We did multivariable analyses for the primary efficacy outcome, including the treatment arm as an independent variable. We entered all variables significantly associated with the outcome on univariate analysis $(\mathrm{P}<0.05)$ and not correlated (Spearman correlation $>0.5$ ) into a logistic regression analysis. We present risk ratios or odds ratios with $95 \%$ confidence intervals.

\section{Results}

We evaluated 782 patients with clinically significant MRSA isolates, of whom 252 were included. The main reasons for exclusion were previous treatment with trimethoprim-sulfamethoxazole or vancomycin for more than 48 hours or inability to provide informed consent,

\begin{tabular}{|c|c|}
\hline \multicolumn{2}{|c|}{ Assessed for eligibility ( $n=782)$} \\
\hline & $\begin{array}{l}\text { Excluded ( } n=530) \text { : } \\
\text { Did not meet inclusion criteria }(n=286) \text { : } \\
\text { Treatment with study drugs }>48 \text { h }(n=164) \\
\text { Allergy }(n=19) \\
\text { Chronic haemodialysis }(n=49) \\
\text { MRSA resistant to trimethoprim-sulfamethoxazole }(n=15) \\
\text { Left sided endocarditis, meningitis, or leukaemia/BMT }(n=28) \\
\text { Participating in another trial }(n=10) \\
\text { Polymicrobial infection excluded }(n=1) \\
\text { Inability to provide informed consent/no legal guardian }(n=165 \\
\text { Declined to participate }(n=79)\end{array}$ \\
\hline \multicolumn{2}{|c|}{ Randomised $(n=252)$} \\
\hline$\downarrow$ & 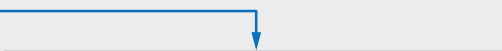 \\
\hline $\begin{array}{l}\text { Allocated to trimethoprim-sulfamethoxazole } \\
(\mathrm{n}=135) \\
\text { Received allocated intervention }(\mathrm{n}=135)\end{array}$ & $\begin{array}{l}\text { Allocated to vancomycin }(n=117) \\
\text { Received allocated intervention }(n=117)\end{array}$ \\
\hline$\downarrow$ & $\downarrow$ \\
\hline $\begin{array}{l}\text { Analysed by intention to treat }(n=135) \text { : } \\
\text { Analysed per protocol }(n=110) \\
\text { Excluded }(n=25) \text { : } \\
\text { Received }<7 \text { days' treatment }(n=24) \\
\text { Post-randomisation exclusion criteria }(n=1)\end{array}$ & $\begin{array}{l}\text { Analysed by intention to treat }(n=117) \text { : } \\
\text { Analysed per protocol }(n=96) \\
\text { Excluded }(n=21) \text { : } \\
\text { Received }<7 \text { days' treatment }(n=16) \\
\text { Post-randomisation exclusion criteria }(n=5)\end{array}$ \\
\hline
\end{tabular}

Flow of patients through study. BMT=bone marrow transplant; MRSA=meticillin resistant Staphylococcus aureus related mostly to severity of sepsis (figure). Inclusion was based on microbiologically documented inclusion criteria in 245 (97\%) patients: 91 (36\%) with bacteraemia and 154 (61\%) with MRSA isolated from other clinical samples. Patients' mean age was 65.8 (SD 17) years, and all infections were healthcare associated (218 (87\%) hospital acquired). Characteristics of patients and infections were mostly balanced between groups; the exception was bacteraemia, which was more common in the vancomycin group (table 1). Appropriate empirical antibiotic treatment was infrequent. Surgical interventions and catheter extraction, when indicated, were performed in similar proportions in the two study groups. Vancomycin trough concentrations were available for $97 / 117(82.9 \%)$ patients in the vancomycin arm $(\geq 10 \mu \mathrm{g} / \mathrm{mL}$ in $80(83 \%)$ patients and $\geq 15 \mu \mathrm{g} / \mathrm{mL}$ in $65(67 \%)$ patients). Isolates' minimum inhibitory concentration to vancomycin was $2 \mu \mathrm{g} / \mathrm{mL}$ in 12/77 (16\%) of patients in the vancomycin arm and lower in the remaining patients. All isolates were susceptible to trimethoprim-sulfamethoxazole (disk zone $\leq 10 \mathrm{~mm}$ ). The median duration of treatment with assigned antibiotics in the per protocol population was 17 (95\% confidence interval 12 to 22) days with trimethoprim-sulfamethoxazole $(\mathrm{n}=110)$ and 14 (13 to 15) days with vancomycin ( $\mathrm{n}=96)$. Potentially effective antibiotics against MRSA (mainly rifampin) were added throughout the treatment course for 14/135 (10\%) patients in the trimethoprim-sulfamethoxazole arm and 8/117 (7\%) patients in the vancomycin $\operatorname{arm}(\mathrm{P}=0.32)$.

We found no significant difference in treatment failure at day 7 between trimethoprim-sulfamethoxazole and vancomycin (risk ratio 1.38, 95\% confidence interval 0.96 to 1.99) (table 2. The failure rate with trimethoprim-sulfamethoxazole was 51/135 (38\%) compared with 32/117 (27\%) with vancomycin, and the 95\% confidence interval for the difference fell outside the lower limit of the $15 \%$ predefined for non-inferiority $(-1.2 \%$ to $21.5 \%)$. Of the components comprising the composite outcome, the advantage to vancomycin emerged from higher bacteraemia persistence at day 7 and lack of improvement in SOFA score at day 7 with trimethoprim-sulfamethoxazole. We observed similar results in the per protocol population of patients completing seven days' treatment (risk ratio 1.24, 0.82 to 1.89 ; absolute difference $9.9 \%,-3.1 \%$ to $22.5 \%$ ) and for patients with bacteraemia (risk ratio 1.40, 0.91 to 2.16). Restricting the analysis to patients in the vancomycin group whose isolates' minimum inhibitory concentrations were below $2 \mu \mathrm{g} / \mathrm{mL}$ resulted in a larger risk ratio in favour of vancomycin (1.64, 0.99 to 2.68).

All cause 30 day mortality did not differ significantly between groups. However, among patients with bacteraemia, mortality was nearly twice as high with trimethoprim-sulfamethoxazole-14/41 (34\%) versus 9/50 (18\%) with vancomycin (risk ratio $1.90,0.92$ to 3.93 ). Among non-bacteraemic patients, mortality rates were low (5/94 (5\%) v 4/67 (6\%)) and not significantly different (risk ratio $0.89,0.25$ to 3.2 ).

We found no significant differences with regard to the pre-defined secondary outcomes (table 2). Persistence 


\begin{tabular}{|c|c|c|c|}
\hline Characteristic & $\begin{array}{l}\text { Trimethoprim- } \\
\text { sulfamethoxazole }(n=135)\end{array}$ & Vancomycin ( $n=117)$ & Pvalue \\
\hline Mean (SD age, years & $64.8(17)$ & $67(17)$ & 0.324 \\
\hline \multicolumn{4}{|l|}{ Functional capacity on admission: } \\
\hline Independent & $75(56)$ & $66(56)$ & \multirow{3}{*}{0.982} \\
\hline Dependent & $31(23)$ & $27(23)$ & \\
\hline Bedridden & $29(21)$ & $24(21)$ & \\
\hline Hospital acquired infection & $115(85)$ & $103(88.0)$ & 0.509 \\
\hline McCabe score, no fatal disease & $104(77)$ & $92(79)$ & 0.761 \\
\hline Mean (SD) Charlson score & $2.59(2.04)$ & $2.65(2.13)$ & 0.829 \\
\hline Diabetes mellitus & $67(50)$ & $54(46)$ & 0.582 \\
\hline Chronic obstructive pulmonary disease & $20(15)$ & $15(13)$ & 0.648 \\
\hline Congestive heart failure, NYHA class III-IV & $29(21)$ & $21(18)$ & 0.483 \\
\hline Chronic renal failure, creatinine $>1.3 \mathrm{mg} / \mathrm{dL}$ & $16(12)$ & $12(10)$ & 0.688 \\
\hline Surgery 30 days before & $64(47)$ & $57(49)$ & 0.835 \\
\hline \multicolumn{3}{|l|}{ Source of MRSA infection: } & \multirow{7}{*}{0.584} \\
\hline Complicated SSTIs & $50(37)$ & $38(32)$ & \\
\hline Bone or joint & $39(29)$ & $32(27)$ & \\
\hline Endovascular & $10(7)$ & $16(14)$ & \\
\hline Pneumonia & $14(10)$ & $14(12)$ & \\
\hline Other & $10(7)$ & $10(9)$ & \\
\hline Primary bacteraemia & $12(9)$ & $7(6)$ & \\
\hline Bacteraemia & $41(30)$ & $50(43)$ & 0.042 \\
\hline \multicolumn{3}{|l|}{ SOFA score at onset of infection: } & \multirow{4}{*}{0.406} \\
\hline 0 & $76(56)$ & $56(48)$ & \\
\hline $1-3$ & $46(34)$ & $47(40)$ & \\
\hline$>3$ & $13(10)$ & $14(12)$ & \\
\hline Mechanically ventilated at onset & $16(12)$ & $11(9)$ & 0.531 \\
\hline Central venous catheter at onset & $17(13)$ & $15(12)$ & 0.957 \\
\hline Mean (SD) creatinine at onset of infection, $\mathrm{mg} / \mathrm{dL}$ & $1.01(0.64)$ & 1.04 (0.71); $(n=116)$ & 0.736 \\
\hline Mean (SD) total leucocytes at onset of infection, $\mathrm{K} / \mathrm{mL}^{3}$ & $11.6(6)$ & 10.5 (4.6); $(n=116)$ & 0.094 \\
\hline Mean (SD) albumin at onset of infection, $\mathrm{mg} / \mathrm{dL}$ & $2.86(0.76) ;(n=108)$ & $2.78(0.63) ;(m=102)$ & 0.392 \\
\hline Polymicrobial infection & $53(39)$ & $41(35)$ & 0.49 \\
\hline Appropriate empirical antibiotic treatment (within 48 h) & $13(10)$ & $14(12)$ & 0.524 \\
\hline Surgery as part of infection management by day $7^{\star}$ & $52 / 71(73)$ & 46/58 (79) & 0.422 \\
\hline Foreign body or catheter removal by day $7 \dagger$ & $13 / 63(21)$ & $15 / 55(27)$ & 0.398 \\
\hline \multicolumn{4}{|c|}{$\begin{array}{l}\text { MRSA=meticillin resistant Staphylococcus aureus; NYHA=New York Heart Association; SOFA=Sequential Organ Failure Assessment; SSTI=skin and sof } \\
\text { tissue infections. } \\
\text { *Denominator is patients in whom surgery was deemed necessary as part of treatment management; among all patients, rates were 52/135 (38.5\%) } \\
\text { versus } 46 / 117 \text { (39.3\%), P=0.9. } \\
\text { †Denominator is patients with central vascular catheter or foreign body. }\end{array}$} \\
\hline
\end{tabular}

of bacteraemia was slightly more common with vancomycin at 48 hours and with trimethoprim-sulfamethoxazole at seven days. There was no significant difference in length of hospital admission for patients discharged alive. Adverse events were reported with similar frequency. Renal failure at day 7 and day 30 was slightly more common with vancomycin, but this was not statistically significant (table 3).

Variables significantly associated with the primary outcome on univariate analysis were included in the multivariable analysis. McCabe score, presence of nasogastric tube or urine catheter at onset of infection, white blood count, and albumin concentrations were associated with treatment failure but correlated with other included variables and were not entered into the regression analysis. Other significant variables are shown in table 4. On multivariable analysis, allocation to trimethoprim-sulfamethoxazole was significantly associated with treatment failure (adjusted odds ratio 2.00, 1.09 to 3.65). Other independent risk factors were bacteraemia and mechanical ventilation at infection onset; surgery in the 30 days before infection was inversely associated with treatment failure.

\section{Discussion}

In a randomised controlled trial including 252 patients with severe MRSA infections, trimethoprim-sulfamethoxazole did not fulfil criteria for non-inferiority to vancomycin. The absolute difference in treatment failure rates at day 7-comprising death, clinical/haemodynamic instability, and persistence of bacteraemia-was $10.4 \%(-1.2 \%$ to $21.5 \%)$ in favour of vancomycin, crossing the upper limit of $15 \%$ difference defined for non-inferiority. After adjustment for differences between groups, treatment with trimethoprim-sulfamethoxazole was significantly associated with treatment failure (odds ratio 2.00, 1.09 to 3.65). Thirty day mortality did not differ significantly between groups (risk ratio 1.27, 0.65 to 2.45); however, among patients with bacteraemia $(n=91)$, death rates were nonsignificantly higher with trimethoprim-sulfamethoxazole. Results were similar in a per protocol analysis. 
Table 2 | Study outcomes. Values are numbers (percentages) unless stated otherwise

\begin{tabular}{|c|c|c|c|c|c|c|}
\hline \multirow[b]{2}{*}{ Outcome } & \multicolumn{3}{|l|}{ All } & \multicolumn{3}{|l|}{ Bacteraemia } \\
\hline & $\begin{array}{l}\text { Trimethoprim- } \\
\text { sulfamethoxazole }\end{array}$ & Vancomycin & $\begin{array}{l}\text { Effect estimate } \\
(95 \% \mathrm{Cl})^{\star}\end{array}$ & $\begin{array}{l}\text { Trimethoprim- } \\
\text { sulfamethoxazole }\end{array}$ & Vancomycin & $\begin{array}{l}\text { Effect estimate } \\
(95 \% \mathrm{Cl})^{\star}\end{array}$ \\
\hline Treatment failure, day 7-ITT $\dagger$ & $51 / 135(38)$ & $32 / 117(27)$ & 1.38 (0.96 to 1.99$)$ & $23 / 41(56)$ & $20 / 50(40)$ & $1.40(0.91$ to 2.16$)$ \\
\hline Treatment failure, day 7-PP† & $37 / 110(34)$ & $26 / 96(27)$ & $1.24(0.82$ to 1.89$)$ & $17 / 33(52)$ & $15 / 42(36)$ & 1.44 (0.85 to 2.44$)$ \\
\hline All cause mortality, 30 days $-\mathrm{ITT} \dagger$ & 19/135 (14) & 13/117 (11) & $1.27(0.65$ to 2.45$)$ & $14 / 41(34)$ & $9 / 50(18)$ & 1.90 (0.92 to 3.93) \\
\hline All cause mortality, 30 days-PPt & $12 / 110(11)$ & 10/96 (10) & 1.05 (0.47 to 2.32$)$ & $9 / 33(27)$ & $6 / 42(14)$ & 1.91 (0.76 to 4.82$)$ \\
\hline Treatment failure or modification $\neq$ & $59 / 135(42)$ & 45/117 (38) & 1.14 (0.84 to 1.53$)$ & 24/41 (59) & $21 / 50(42)$ & 1.39 (0.92 to 2.11) \\
\hline Bacteraemia duration $>48 \mathrm{~h}$ & $11 / 135(8)$ & 15/117 (13) & 0.64 (0.3 to 1.33$)$ & $11 / 41(27)$ & $15 / 50(30)$ & 0.89 (0.46 to 1.73$)$ \\
\hline Bacteriological failure, day 7 & $10 / 135(7)$ & 4/117 (3) & 2.17 (0.7 to 6.73$)$ & $6 / 41(15)$ & 4/50 (8) & 1.83 (0.55 to 6.05$)$ \\
\hline Hospital admission duration§ & $\begin{array}{l}14 \text { (11.6 to } 16.4) ; \\
(n=117)\end{array}$ & $\begin{array}{l}15 \text { (14.5 to } 15.5) \\
(n=102)\end{array}$ & $-1(-4.36$ to 2.36$)$ & $\begin{array}{l}15 \text { (7.1 to } 22.9) ; \\
(n=29)\end{array}$ & $\begin{array}{l}18 \text { (8.6 to } 27.4) ; \\
(n=39)\end{array}$ & $-3(-11.62$ to 5.62$)$ \\
\hline Fever duration§ & $1(0.5$ to 1.5$) ;(n=132)$ & 1 (1 to 1$) ;(n=114)$ & $0(-0.49$ to 0.49$)$ & 2 (1.5 to 2.5$) ;(n=40)$ & 2 (1 to 3$) ;(n=48)$ & $0(-0.7$ to 0.7$)$ \\
\hline Resistance development $ף$ & $5 / 135(4)$ & $6 / 117(5)$ & 0.72 (0.23 to 2.31) & - & - & - \\
\hline \multicolumn{7}{|c|}{$\begin{array}{l}\text { *Risk ratios }(95 \% \mathrm{Cl}) \text { presented for categorical variables and median differences }(95 \% \mathrm{Cl}) \text { for continuous outcomes. } \\
\text { †ITT=intention to treat; } \mathrm{PP}=\text { per protocol analysis; all other outcomes reported by intention to treat. } \\
\text { fFailure at day } 7 \text { or deviation from assigned regimen in first seven days of treatment. } \\
\text { \$Reported as median }(95 \% \mathrm{CI}) \text { days. Hospital admission duration reported only for patients discharged alive. }\end{array}$} \\
\hline
\end{tabular}

Bacteriological cure and adverse event rates did not differ significantly between groups.

Our trial was pragmatic, targeting all patients treated with vancomycin in clinical practice. We defined no exclusion criteria related to severity of background illness or sepsis. Exclusions were based only on contraindications for trimethoprim-sulfamethoxazole (such as chronic haemodialysis) or previous evidence suggesting inferiority of trimethoprim-sulfamethoxazole. We thus excluded left sided endocarditis owing to an in vivo study of endocarditis in rabbits showing lower survival and vegetation sterilisation and higher bacterial load on vegetations with trimethoprim-sulfamethoxazole compared with vancomycin and other antibiotics. ${ }^{32}$ We excluded patients with MRSA meningitis owing to inferiority of trimethoprim-sulfamethoxazole to nafcillin in an experimental model of meticillin susceptible $S$ aureus meningitis and lack of evidence in MRSA, despite good penetration of trimethoprim-sulfamethoxazole to the brain. ${ }^{33}{ }^{34}$ We originally intended to recruit patients with non-microbiologically documented,

\begin{tabular}{|c|c|c|c|}
\hline Adverse event & $\begin{array}{l}\text { Trimethoprim- } \\
\text { sulfamethoxazole }\end{array}$ & Vancomycin & Risk ratio $(95 \% \mathrm{Cl})$ \\
\hline Any & 39/132 (30) & $26 / 115(23)$ & 1.31 (0.85 to 2.01$)$ \\
\hline Requiring discontinuation & 14/132(11) & $8 / 115(7)$ & 1.52 (0.66 to 3.5$)$ \\
\hline RIFLE day $7^{\star}$ : & $(n=108)$ & $(n=100)$ & \\
\hline None & $90(83)$ & $86(86)$ & Reference \\
\hline Risk & $7(6)$ & $2(2)$ & 3.18 (0.68 to 14.88$)$ \\
\hline Injury & $11(10)$ & $8(8)$ & 1.28 (0.54 to 2.04$)$ \\
\hline Failure & 0 & $4(4)$ & Not assessed \\
\hline RIFLE risk, injury, or failure, day $7^{\star}$ & $18 / 108(17)$ & $14 / 100(14)$ & $1.19(0.63$ to 2.26$)$ \\
\hline RIFLE day $30^{*}:$ & $(n=87)$ & $(n=86)$ & \\
\hline None & $75(86)$ & $68(79)$ & Reference \\
\hline Risk & $2(2)$ & $4(5)$ & 0.47 (0.09 to 2.48$)$ \\
\hline Injury & $3(3)$ & $10(12)$ & 0.3 (0.09 to 1.05$)$ \\
\hline Failure & $7(8)$ & $4(5)$ & $1.54(0.47$ to 5.04$)$ \\
\hline RIFLE risk, injury, or failure, day $30^{\star}$ & $12 / 87(14)$ & $18 / 86(21)$ & 0.66 (0.34 to 1.28$)$ \\
\hline Any rash & 12/132 (9) & $12 / 115$ (104) & $0.87(0.41$ to 1.86$)$ \\
\hline Diarrhoeat & 12/132(9) & $11 / 115(10)$ & 0.95 (0.44 to 2.07 ) \\
\hline
\end{tabular}

highly probable MRSA infections. However, in practice, we found identifying such patients prospectively difficult and practically the trial included only patients with microbiologically confirmed MRSA infections. The main reason precluding inclusion of patients was inability to obtain informed consent at the time of acute sepsis. In Israel, regulations allow only legal guardians to replace patients' informed consent (informed consent from relatives is not allowed), and with acute conditions most patients did not have an appointed legal guardian.

\section{Comparison with other studies}

Few randomised controlled trials have assessed the effectiveness of trimethoprim-sulfamethoxazole for the treatment of staphylococcal infections. A single randomised controlled trial compared trimethoprim-sulfamethoxazole against vancomycin for staphylococcal infections. ${ }^{23}$ The trial randomised 228 intravenous drug users with suspected staphylococcal infections and analysed 101 with proven infections (54

\begin{tabular}{|c|c|c|}
\hline \multirow[b]{2}{*}{ Variable } & \multicolumn{2}{|c|}{$\begin{array}{l}\text { Treatment failure at day } 7 \text { : odds ratio } \\
(95 \% \mathrm{Cl})^{*}\end{array}$} \\
\hline & Univariate & Multivariate \\
\hline Age & 1.02 (1 to 1.03$)$ & 1.01 (0.99 to 1.03 ) \\
\hline Charlson score & 1.24 (1.09 to 1.42$)$ & 1.15 (0.99 to 1.35$)$ \\
\hline Bedriddent & 1.96 (1.06 to 3.65$)$ & $0.80(0.36$ to 1.76$)$ \\
\hline $\begin{array}{l}\text { Chronic obstructive } \\
\text { pulmonary disease }\end{array}$ & $2.16(1.05$ to 4.45$)$ & 0.76 (0.30 to 1.90$)$ \\
\hline Previous operation $\neq$ & 0.49 (0.28 to 0.83$)$ & $0.52(0.28$ to 0.96$)$ \\
\hline Mechanical ventilationt & 5.98 (2.49 to 14.3$)$ & $5.02(1.62$ to 15.6$)$ \\
\hline SOFA scoret & $1.41(1.2$ to 1.66$)$ & $1.15(0.94$ to 1.40$)$ \\
\hline Bacteraemia & 2.71 (1.57 to 4.67$)$ & 2.24 (1.20 to 4.18$)$ \\
\hline Trimethoprim- & 1.61 (0.94 to 2.75$)$ & 2.00 (1.09 to 3.65$)$ \\
\hline
\end{tabular}

SOFA=Sequential Organ Failure Assessment.

*Hosmer-Lemeshow $\mathrm{P}=0.08$; area under receiver operating

characteristics curve 0.75 ( $95 \% \mathrm{Cl} 0.68$ to 0.82 ).

+Variables documented at infection onset; SOFA analysed as continuous variable.

$\neq$ In 30 days before infection. 
with meticillin susceptible $S$ aureus and 47 with MRSA). Vancomycin was superior to trimethoprim-sulfamethoxazole among patients with meticillin susceptible $S$ aureus infections, but no differences were observed for patients with MRSA. Specifically, bacteraemia duration (total 57 patients) was longer with trimethoprim-sulfamethoxazole treatment for meticillin susceptible $S$ aureus ( $7.8 \vee 3.5$ days; $\mathrm{P}=0.05$ ) and similar for MRSA (5.2 $v 5.6$ days). The authors concluded that trimethoprim-sulfamethoxazole is a valuable alternative for the treatment of MRSA infections, unlike the conclusions of our study. However, the study included significantly younger and less ill patients, with a single death compared with the $13 \%$ mortality rate in our study. In a recent randomised controlled trial, trimethoprim-sulfamethoxazole combined with rifampin was compared with linezolid in the treatment of MRSA infections (bacteraemia in 18/150 (12\%) and mortality in 14/150 (9\%)), showing no differences in failure/relapse rates or mortality. ${ }^{15}$ Among 50 patients with chronic meticillin susceptible $S$ aureus osteomyelitis randomised to trimethoprim-sulfamethoxazole-rifampin versus oxacillin, long term outcomes were similar and patients allocated to trimethoprim-sulfamethoxazole-rifampin had a significantly shorter hospital stay (median $51 v 31$ days). ${ }^{35}$ Finally, trimethoprim-sulfamethoxazole was not significantly different from doxycycline for uncomplicated skin and soft tissue infections in a small randomised controlled trial assessing 22 patients with MRSA. ${ }^{36}$

The main treatment options for MRSA infections other than vancomycin (or teicoplanin) and trimethoprim-sulfamethoxazole include linezolid, tedizolid, telavancin, dalbavancin, oritavancin, daptomycin, tigecycline, and ceftaroline. ${ }^{37}$ Most of the new antibiotics were evaluated for the treatment of skin and soft tissue infections in randomised controlled trials. Mortality was rarely assessed in these trials and when reported was very low (usually $<1 \%$ ). Few randomised controlled trials targeted infections other than skin and soft tissue infections, and most did not include only MRSA. Daptomycin was non-inferior to standard therapy in a randomised controlled trial including 245 patients with $S$ aureus bacteraemia, of whom 89 had MRSA bacteraemia and were treated with daptomycin or vancomycin. ${ }^{38}$ The overall mortality was $11 \%$. Similar efficacy and safety for was shown for linezolid and vancomycin in nosocomial pneumonia, and the mortality rate in these trials was $14 \% .{ }^{39}$ Tigecycline was non-inferior to vancomycin in a randomised controlled trial including 156 patients with invasive MRSA infections, but most were skin and soft tissue infections (mortality rate 5\%). ${ }^{40}$ Telavancin was non-inferior to vancomycin in hospital acquired pneumonia (293 patients with MRSA, overall mortality in the study 19\%). ${ }^{41}$ Compared with these trials, ours included a larger group of patients with MRSA bacteraemia and a sicker cohort overall, as evidenced by the mortality rates, more closely reflecting patients treated with MRSA infections in clinical practice.

\section{Limitations of study}

The main limitation of our trial is the small sample size of patients with bacteraemia, in whom results suggest an important advantage for vancomycin. No statistically significant differences were observed between groups at baseline for nearly all variables. However, a subtle difference existed for important prognostic variables, and more patients allocated to vancomycin were bacteraemic. Multivariable analysis of the entire study cohort showed a larger advantage of vancomycin with regard to treatment failure, reaching statistical significance. Patients with polymicrobial infections and receiving additional antibiotics were included in the study, reflecting its pragmatic nature. However, these were mostly inactive against MRSA and concomitant antibiotics covering MRSA were not allowed at randomisation. Patients excluded owing to inability to provide informed consent had higher severity of illness scores than those included, affecting the trial's external validity. Finally, the dosing of vancomycin and trough levels achieved ( $\geq 15 \mu \mathrm{g} / \mathrm{mL}$ in only $67 \%$ of patients) were lower than currently recommended, ${ }^{20}$ potentially resulting in an underestimation of the efficacy of vancomycin and the difference between arms.

\section{Conclusion}

Trimethoprim-sulfamethoxazole did not achieve non-inferiority compared with vancomycin among patients with invasive MRSA infections. In the subgroup of patients with bacteraemia, the difference in treatment failure and all cause mortality might be clinically important. Trimethoprim-sulfamethoxazole should not be used for the treatment of severe MRSA infections. We propose a further randomised controlled trial to examine the feasibility of step-down from vancomycin to trimethoprim-sulfamethoxazole, allowing early discharge of patients with MRSA responding to treatment.

Contributors: MP and JB contributed equally. MP, JB, and LL contributed to study conception and design and analysed the data. All authors were involved in data collection, as were Maria Raskin, Ynon Lischinski, Morad Wattad, Olga Talker, Rawi Hazan, Laura Farbman, Vered Shani, and Vered Zaretsky (Rabin Medical Center); and Roni Bitterman, Anat Stern, Yael Dishon-Benattar, and Fidi Koppel (Rambam Health Care Campus). MP drafted the manuscript. All authors were involved in data interpretation and approval of the final manuscript. $M P$ and $L L$ are the guarantors.

Funding: The study was partially funded by the Sima Lior scholarship 2008 (MP) and Rabin Medical Center Young Investigator scholarship 2009 (DY). The funders had no role in trial design; data collection, analysis, or interpretation; or manuscript preparation.

Competing interests: All authors have completed the ICMJE uniform disclosure form at www.icmje.org/coi_disclosure.pdf (available on request from the corresponding author) and declare: no support from any organisation for the submitted work other than that detailed above; no financial relationships with any organisations that might have an interest in the submitted work in the previous three years; no other relationships or activities that could appear to have influenced the submitted work.

Ethical approval: The trial was approved by the ethics committees in each study centre, and informed consent was obtained from all patients or their legal guardian.

Transparency declaration: The lead authors (the manuscript's guarantors) affirm that the manuscript is an honest, accurate, and transparent account of the study being reported; that no important aspects of the study have been omitted; and that any discrepancies from the study as planned have been explained. 
Data sharing: Case report forms, dataset, and statistical code are available from the corresponding author.

This is an Open Access article distributed in accordance with the Creative Commons Attribution Non Commercial (CC BY-NC 4.0) license, which permits others to distribute, remix, adapt, build upon this work non-commercially, and license their derivative works on different terms, provided the original work is properly cited and the use is non-commercial. See: http://creativecommons.org/licenses/ by-nc/4.0/.

1 Elwell LP, Wilson HR, Knick VB, Keith BR. In vitro and in vivo efficacy of the combination trimethoprim-sulfamethoxazole against clinical isolates of methicillin-resistant Staphylococcus aureus. Antimicrob Agents Chemother 1986;29:1092-4

2 Nathwani D, Davey PG, Marwick CA. MRSA: treating people with infection. BMJ Clin Evid 2010:2010:pii:0922.

3 Pappas G, Athanasoulia AP, Matthaiou DK, Falagas ME. Trimethoprimsulfamethoxazole for methicillin-resistant Staphylococcus aureus: a forgotten alternative? J Chemother 2009;21:115-26.

4 Stryjewski ME, Chambers HF. Skin and soft-tissue infections caused by community-acquired methicillin-resistant Staphylococcus aureus. Clin Infect Dis 2008;46(suppl 5):S368-77.

5 Richter SS, Heilmann KP, Dohrn CL, Riahi F, Costello AJ, Kroeger JS, et al. Activity of ceftaroline and epidemiologic trends in Staphylococcus aureus isolates collected from 43 medical centers in the United States in 2009. Antimicrob Agents Chemother 2011;55:4154-60.

6 Wackett A, Nazdryn A, Spitzer E, Singer AJ. MRSA rates and antibiotic susceptibilities from skin and soft tissue cultures in a suburban ED. J Emerg Med 2012;43:754-7.

7 Wood JB, Smith DB, Baker EH, Brecher SM, Gupta K. Has the emergence of community-associated methicillin-resistant Staphylococcus aureus increased trimethoprim-sulfamethoxazole use and resistance? A 10-year time series analysis. Antimicrob Agents Chemother 2012;56:5655-60.

8 Campbell ML, Marchaim D, Pogue IM, Sunkara B, Bheemreddy S, Bathina P, et al. Treatment of methicillin-resistant Staphylococcus aureus infections with a minimal inhibitory concentration of $2 \mathrm{mug} /$ $\mathrm{mL}$ to vancomycin: old (trimethoprim/sulfamethoxazole) versus new (daptomycin or linezolid) agents. Ann Pharmacother 2012;46:1587-97.

9 Muileboom J, Hamilton M, Parent K, Makahnouk D, Kirlew M, Saginur R, et al. Community-associated methicillin-resistant Staphylococcus aureus in northwest Ontario: a five-year report of incidence and antibiotic resistance. Can J Infect Dis Med Microbiol 2013;24:e42-4.

10 Levesque S, Bourgault AM, Galarneau LA, Moisan D, Doualla-Bell F, Tremblay C. Molecular epidemiology and antimicrobial susceptibility profiles of methicillin-resistant Staphylococcus aureus blood culture isolates: results of the Quebec Provincial Surveillance Programme. Epidemiol Infect 2015;143:1511-8.

11 Zhanel GG, Adam HJ, Baxter MR, Fuller J, Nichol KA, Denisuik AJ, et al. Antimicrobial susceptibility of 22746 pathogens from Canadian hospitals: results of the CANWARD 2007-11 study. J Antimicrob Chemother 2013;68(suppl 1):i7-22

12 Hanaki H, Cui L, Ikeda-Dantsuji Y, Nakae T, Honda J, Yanagihara K, et al. Antibiotic susceptibility survey of blood-borne MRSA isolates in Japan from 2008 through 2011. J Infect Chemother 2014:20:527-34

13 Farrell DJ, Flamm RK, Sader HS, Jones RN. Ceftobiprole activity against over 60,000 clinical bacterial pathogens isolated in Europe, Turkey, and Israel from 2005 to 2010. Antimicrob Agents Chemother 2014; $58: 3882-8$.

14 Schaumburg F, Idelevich EA, Peters G, Mellmann A, von Eiff C, Becker $\mathrm{K}$. Trends in antimicrobial non-susceptibility in methicillin-resistant Staphylococcus aureus from Germany (2004-2011). Clin Microbiol Infect 2014;20:0554-7.

15 Harbarth S, von Dach E, Pagani L, Macedo-Vinas M, Huttner B, Olearo F, et al. Randomized non-inferiority trial to compare trimethoprim/ sulfamethoxazole plus rifampicin versus linezolid for the treatment of MRSA infection. J Antimicrob Chemother 2015;70:264-72.

16 Australian Group on Antimicrobial Resistance (AGAR). SAP 2011 antimicrobial susceptibility report. www.agargroup.org/files/SAP\%20 2011\%20Susceptibility\%20Report\%20Final.pdf.

17 Australian Group on Antimicrobial Resistance (AGAR). SAP 2012 antimicrobial susceptibility report. www.agargroup.org/files/ Staphylococcus\%20aureus\%20Programme\%202012\%20 Susceptibility\%20Report.pdf.

18 Nurjadi D, Olalekan AO, Layer F, Shittu AO, Alabi A, Ghebremedhin B, et al. Emergence of trimethoprim resistance gene dfrG in Staphylococcus aureus causing human infection and colonization in sub-Saharan Africa and its import to Europe. J Antimicrob Chemother 2014;69:2361-8

19 Sharma NK, Garg R, Baliga S, Bhat KG. Nosocomial infections and drug susceptibility patterns in methicillin sensitive and methicillin resistant Staphylococcus aureus. J Clin Diagn Res 2013;7:2178-80

20 Kali A, Stephen S, Umadevi S, Kumar S, Joseph NM, Srirangaraj S. Changing trends in resistance pattern of methicillin resistant Staphylococcus aureus. J Clin Diagn Res 2013;7:1979-82.

21 Liu C, Bayer A Cosgrove SE, Daum RS, Fridkin SK, Gorwitz RJ et al. Clinical practice guidelines by the Infectious Diseases Society of America for the treatment of methicillin-resistant Staphylococcus aureus infections in adults and children: executive summary. Clin Infect Dis 2011:52:285-92.

$22 \mathrm{Kim} \mathrm{SH}$, Kim KH, Kim HB, Kim NJ, Kim EC, Oh MD, et al. Outcome of vancomycin treatment in patients with methicillin-susceptible Staphylococcus aureus bacteremia. Antimicrob Agents Chemother 2008;52:192-7.

23 Markowitz N, Quinn EL, Saravolatz LD. Trimethoprimsulfamethoxazole compared with vancomycin for the treatment of Staphylococcus aureus infection. Ann Intern Med 1992:117:390-8.

24 Holland TL, Arnold C, Fowler VG Jr. Clinical management of Staphylococcus aureus bacteremia: a review. JAMA 2014;312:1330-41.

25 Goldberg E, Paul M, Talker O, Samra Z, Raskin M, Hazzan R, et al. Co-trimoxazole versus vancomycin for the treatment of methicillinresistant Staphylococcus aureus bacteraemia: a retrospective cohor study. I Antimicrob Chemother 2010;65:1779-83.

26 Horan TC, Andrus M, Dudeck MA. CDC/NHSN surveillance definition of health care-associated infection and criteria for specific types of infections in the acute care setting. Am I Infect Control 2008;36:309-32.

27 Bone RC, Fisher CJ Jr, Clemmer TP, Slotman GJ, Metz CA, Balk RA. Sepsis syndrome: a valid clinical entity. Crit Care Med 1989;17:389-93.

28 Carnicer-Pont D, Bailey KA, Mason BW, Walker AM, Evans MR, Salmon RL. Risk factors for hospital-acquired methicillin-resistant Staphylococcus aureus bacteraemia: a case-control study. Epidemiol Infect 2006;134:1167-73.

29 Spicehandler J, Pollock AA, Simberkoff MS, Rahal JJ Jr. Intravenous pharmacokinetics and in vitro bactericidal activity of trimethoprimsulfamethoxazole. Rev Infect Dis 1982;4:562-5.

30 Bellomo R, Kellum JA, Ronco C. Defining and classifying acute rena failure: from advocacy to consensus and validation of the RIFLE criteria. Intensive Care Med 2007:33:409-13.

31 Bonett DG, Price RM. Statistical inference for a linear function of medians: confidence intervals, hypothesis testing, and sample size requirements. Psychol Methods 2002;7:370-83.

32 De Gorgolas M, Aviles P, Verdejo C, Fernandez Guerrero ML. Treatment of experimental endocarditis due to methicillin-susceptible or methicillinresistant Staphylococcus aureus with trimethoprim-sulfamethoxazole and antibiotics that inhibit cell wall synthesis. Antimicrob Agents Chemother 1995;39:953-7.

33 Scheld WM, Keeley JM, Field MR, Brodeur JP. Co-trimoxazole versus nafcillin in the therapy of experimental meningitis due to Staphylococcus aureus. J Antimicrob Chemother 1987;19:647-58.

34 Levitz RE, Quintiliani R. Trimethoprim-sulfamethoxazole for bacterial meningitis. Ann Intern Med 1984;100:881-90.

35 Euba G, Murillo O, Fernandez-Sabe N, Mascaro J, Cabo J, Perez A, et al Long-term follow-up trial of oral rifampin-cotrimoxazole combination versus intravenous cloxacillin in treatment of chronic staphylococcal osteomyelitis. Antimicrob Agents Chemother 2009:53:2672-6.

36 Cenizal MJ, Skiest D, Luber S, Bedimo R, Davis P, Fox P, et al. Prospective randomized trial of empiric therapy with trimethoprimsulfamethoxazole or doxycycline for outpatient skin and soft tissue infections in an area of high prevalence of methicillin-resistant Staphylococcus aureus. Antimicrob Agents Chemother 2007; 51:2628-30.

37 Rodvold KA, McConeghy KW. Methicillin-resistant Staphylococcus aureus therapy: past, present, and future. Clin Infect Dis 2014;58(suppl 1):S20-7.

38 Fowler VG Jr, Boucher HW, Corey GR, Abrutyn E, Karchmer AW, Rupp $M E$, et al. Daptomycin versus standard therapy for bacteremia and endocarditis caused by Staphylococcus aureus. N Engl/ Med 2006;355:653-65.

39 Kalil AC, Klompas M, Haynatzki G, Rupp ME. Treatment of hospitalacquired pneumonia with linezolid or vancomycin: a systematic review and meta-analysis. BMJ Open 2013;3:e003912.

40 Florescu I, Beuran M, Dimov R, Razbadauskas A, Bochan M, Fichev G, et al. Efficacy and safety of tigecycline compared with vancomycin or linezolid for treatment of serious infections with methicillin-resistant Staphylococcus aureus or vancomycin-resistant enterococci: a phase 3 , multicentre, double-blind, randomized study. J Antimicrob Chemother 2008;62(suppl 1):i17-28.

41 Rubinstein E, Lalani T, Corey GR, Kanafani ZA, Nannini EC, Rocha MG, et al. Telavancin versus vancomycin for hospital-acquired pneumonia due to gram-positive pathogens. Clin Infect Dis 2011;52:31-40.

C BMJ Publishing Group Ltd 2015 